\title{
BMJ Open Care of the patient with invasive meningococcal disease by prehospital emergency medical service clinicians: a scoping review
}

\author{
James Pearce (D) , ${ }^{1,2}$ Micah Peters, ${ }^{3}$ Nikki May, ${ }^{4}$ Helen Marshall (D) , ${ }^{5,6}$ \\ Cindy Hein, ${ }^{1,2}$ Hugh Grantham ${ }^{2,7}$
}

To cite: Pearce J, Peters M, May N, et al. Care of the patient with invasive meningococcal disease by prehospital emergency medical service clinicians: a scoping review. BMJ Open 2020;10:e033447. doi:10.1136/ bmjopen-2019-033447

- Prepublication history and additional material for this paper are available online. To view these files, please visit the journal online (http://dx.doi. org/10.1136/bmjopen-2019033447).

Received 06 August 2019 Revised 12 November 2019 Accepted 12 February 2020

Check for updates

(C) Author(s) (or their employer(s)) 2020. Re-use permitted under CC BY-NC. No commercial re-use. See rights and permissions. Published by BMJ.

For numbered affiliations see end of article.

Correspondence to

Mr James Pearce;

james.pearce@flinders.edu.au

\section{ABSTRACT}

Objective The objective of this scoping review is to systematically map the literature to identify the scope, depth, key concepts and gaps in the evidence regarding care of the patient with invasive meningococcal disease by emergency medical service (EMS) clinicians.

Design Scoping review. This review is reported in accordance with the Preferred Reporting Items for Systematic Reviews and Meta-Analyses Extension for Scoping Reviews reporting guideline.

Eligibility criteria Sources which focused on patients with invasive meningococcal disease (population), where the care of EMS clinicians was the focus (concept), in EMS systems worldwide (context) were eligible for inclusion. Search strategy This review utilised a comprehensive search strategy including MEDLINE, Embase, Emcare, CINAHL, Scopus, Web of Science, Google Scholar and 'grey' literature databases from 1992 to January 2019. The search also included a Google search, a hand-search of relevant journals, screening of reference lists, contact with authors of included sources and use of social media in an attempt to locate all sources of evidence which fit the inclusion criteria of the review. Two reviewers independently screened sources for inclusion.

Results The search yielded 1803 unique records, of which 10 were included in the synthesis. No original research papers were identified, with all sources classed as either clinical audit or text and opinion literature. The dominant concept throughout the literature is that early antibiotic therapy is critical in the treatment of invasive meningococcal disease.

Conclusions Overall, there is a very narrow scope and shallow depth of literature on the topic of interest. There are gaps in the evidence regarding the care of the patient with invasive meningococcal disease by EMS clinicians. Despite these shortfalls, current consensus-based guidelines should direct clinical practice. Further research is planned to bridge the gaps in knowledge to support best practice.

\section{INTRODUCTION}

\section{Rationale}

Invasive meningococcal disease (IMD), caused by Neisseria meningitidis, is one of the most rapidly progressive infectious diseases.
Strengths and limitations of this study

- The review utilised a best-practice approach to the conduct and reporting of scoping reviews; the Preferred Reporting Items for Systematic Reviews and Meta-Analyses Extension for Scoping Reviews.

- A comprehensive search strategy was designed by a research librarian with expertise in systematic reviews and includes a novel search technique using social media.

- Relevant non-English studies were translated and included, reducing geographical and structural biases.

- The review team consists of experts in scoping review methodology, literature searching, meningococcal disease and emergency medical services.

- There was no primary research located in this review.

Despite advances in clinical management, it still carries a significant burden of disease worldwide. ${ }^{1}$ IMD remains a significant public health concern due to the sudden onset, unpredictable clinical progress and often rapid clinical course. Due to the fulminant nature of IMD, it is important that early identification and appropriate management are provided by emergency medical service (EMS) clinicians to reduce morbidity and mortality, and ensure the best patient outcomes. A comprehensive background and rationale for this review can be found in the published review protocol. ${ }^{2}$

\section{Preliminary literature search}

A preliminary search of the International Prospective Register of Systematic Reviews, Open Science Framework, Epistemonikos, PubMed, JBI Database of Systematic Reviews and Implementation Reports, and The Cochrane Database of Systematic Reviews did not locate any existing or underway systematic reviews or scoping reviews that address 
the aim of this review. Two published systematic reviews investigating the effectiveness of pre-admission antibiotics for IMD were located but did not include any EMS data. $^{34}$ Three published systematic reviews investigating EMS identification or management of sepsis were located but did not explicitly discuss IMD. ${ }^{5-7}$ Preliminary literature searches also identified that there appeared to be a general scarcity of primary evidence sources in this field indicating that a scoping review may be a warranted as an appropriate approach for identifying and assessing any existing evidence.

\section{The scoping review approach}

Scoping reviews can be conducted to identify the range, nature and extent of the evidence on a topic or question. ${ }^{8}$ They are used to map the literature, collate the evidence on a topic and summarise the characteristics and findings of the sources of evidence. Scoping review methodology is particularly useful where there is a body of literature which has not yet been comprehensively reviewed ${ }^{9}$ such as in the case of the present review that seeks to explore the characteristics and overall parameters of what has been studied in this field. The first framework for conducting scoping reviews was published by Arksey and O'Malley in $2015^{10}$ and has been extended on and refined several times since. ${ }^{811-13}$

\section{Objective}

Underpinned by the review question, 'What is the state of evidence regarding the care of patients with IMD by EMS clinicians in the international literature?', the objective of this scoping review was to systematically map the literature to identify the scope, depth and key concepts in the evidence, and to identify gaps in evidence regarding the care of patients with IMD by EMS clinicians. The purpose of the review is to present the current state of evidence, inform clinical practice and guideline development, and identify gaps in the evidence requiring further research.

\section{METHODS}

This scoping review followed the methods described by Peters and colleagues, ${ }^{13}$ and the Joanna Briggs Institute (JBI),${ }^{14}$ and is reported in accordance with the Preferred Reporting Items for Systematic Reviews and Meta-Analyses Extension for Scoping Reviews (PRISMA-ScR) reporting guideline. ${ }^{8}$ A completed PRISMA-ScR checklist has been included as online supplementary file 1 .

\section{Protocol and registration}

An a priori protocol for this review was published in a peerreviewed journal. ${ }^{2}$ The protocol manuscript was prepared in accordance with the PRISMA-Protocols reporting guideline. ${ }^{15}$ The protocol was disseminated throughout the extensive professional networks of the author group, including via social media and at an international paramedic research symposium to solicit feedback. The protocol was also registered prospectively with the Open
Science Framework ${ }^{16}$ on 25 September 2018 (https:// osf.io/ubd7w/), and was updated on 7 December 2018 following several minor updates (https://osf.io/z639u/).

\section{Eligibility criteria}

Any published or unpublished ('grey') sources which aligned with the research objective were eligible for inclusion. Because of the scarcity of literature in this specific field, text and opinion literature including expert opinion, commentaries, reviews and narratives were also eligible for inclusion, as these constituted the best available evidence. ${ }^{17}$ The starting search date for papers was 1992, which was the year of publication of several of the seminal papers investigating the use of antibiotics for IMD in the community setting. ${ }^{18-22}$ There were no language restrictions on the search or screening processes; Google Translate was used to perform a translation of the title and abstract of any sources if they were located in the searches and appeared to possibly fit the inclusion criteria. Cochrane's TaskExchange was used where complete translations of full-text articles were required. This review follows the population, concept, context inclusion criteria format. ${ }^{13}{ }^{14}$

\section{Population}

This review included literature that focused on human subjects of any age, gender, ethnicity, pregnancy status or comorbidities with suspected or confirmed IMD. This included patients with meningococcal septicaemia, meningococcal meningitis and mixed clinical presentations, as defined by the individual sources. Sources which reported on patients with suspected or confirmed sepsis/septicaemia from causes other than IMD were not included. This is because IMD has a clinical course which is distinct from other causes of sepsis (such as sepsis from a urinary or pulmonary origin).

\section{Concept}

This review included sources that reported on any aspect of the care of IMD by EMS clinicians, which may broadly be categorised as either identification or management. Identification included but was not limited to factors regarding the identification, assessment, recognition, clinical judgement or provisional diagnosis relating to IMD, such as screening tools/items, clinical history, risk factors, or the presence or absence of certain signs and symptoms such as a rash. Management included but was not limited to clinical decision-making, oxygen therapy, fluid therapy, vasopressors, the administration of antibiotics, adjunct therapies (eg, paracetamol or steroids), hospital pre-alert notifications and disposition choices.

\section{Context}

The context of this review was the EMS, which may also be known as an ambulance service or paramedic service. This scoping review included sources that reported on EMS clinicians including but not limited to paramedics, nurses, medical practitioners and the various levels of ambulance technician (eg, first responders, emergency medical 
technicians or ambulance officers) delivering patient care in EMS systems. EMS systems which operate either Franco-German, Anglo-American, Basic Life Support (BLS) or Advanced Life Support structures as defined by $\mathrm{Al}-\mathrm{Shaqsi}{ }^{23}$ were included in this scoping review. Literature which reported on in-hospital care, including emergency department or intensive care/therapy unit care, or non-EMS prehospital care (eg, general/family practice) were not included in this review, as these specific contexts have been studied and reviewed elsewhere.

\section{Excluded sources}

EMS clinical practice guidelines (CPGs) pertaining to IMD were not included in this review as they will be described, compared and comprehensively analysed using the Appraisal of Guidelines for Research and Evaluation II instrument ${ }^{24}$ (a tool to assess the methodological quality of CPGs) in separate future publications. Textbooks, social media posts, blogs and websites were not included in this review.

\section{Information sources}

For published literature, the following databases were searched from 1992 to January 2019: MEDLINE (OVID interface), Embase (OVID interface), Emcare (OVID interface), CINAHL Complete (EBSCO), Scopus and Web of Science. To ensure comprehensiveness, a search of Google Scholar was also carried out. ${ }^{25} 26$ The Google Scholar search was limited to the first 200 results, sorted by relevance.

Including 'grey' literature in the search strategy helps minimise the risk of publication bias. ${ }^{27} \mathrm{~A}$ search for theses was carried out in ProQuest Dissertations and Theses, Open Thesis, Trove and the Networked Library of Theses and Dissertations. Clinical trial registries were also included to identify completed and ongoing clinical studies. ${ }^{28}$ Included databases were as follows: ClinicalTrials.gov, Central Register of Controlled Trials, WHO International Clinical Trials Registry Platform and ISRCTN. A Google search was also undertaken using the Advanced Search function. To avoid personalisation of search results, searches were performed after a fresh install of a new browser, limited to the first 200 results, ordered by relevance.

As electronic database searches may not reveal all possible literature on a topic, ${ }^{29}$ to ensure that all possible sources were included in the review, a hand-search (limited to the last 10 years due to practical time constraints) was also undertaken in the following peer-reviewed journals of high relevance to EMS:

- Australasian Journal of Paramedicine (formerly known as the Journal of Emergency Primary Healthcare)

- British Paramedic Journal

- International Paramedic Practice

- Irish Journal of Paramedicine

- Journal of Paramedic Practice

- Prehospital and Disaster Medicine

- Prehospital Emergency Care
James Pearce @James_W_Pearce ·Jan 8

Can you help? A scoping review on the care of a patient with invasive

\#meningococcal disease by \#EMS clinicians. Part of research to combat this deadly disease. Please RT \& tag any known literature to @James_W_Pearce.

Thanks! \#sepsis \#paramedic \#systematicreview \#scopingreview

Care of the patient with

invasive meningococcal disease

by EMS clinicians: a scoping review

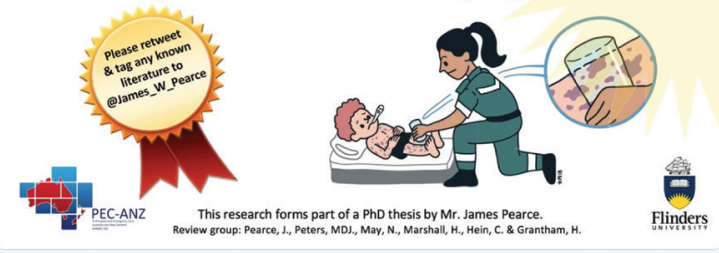

Figure 1 Scoping review Tweet.

The corresponding authors of all sources of evidence included in the review were contacted, reference lists of all included sources were scanned, and social media was used in an attempt to identify any potential additional sources.

\section{Social media search}

In addition to the electronic database searches, handsearch and direct contact with authors, the lead author (JP) posted on Twitter requesting users to identify any known relevant literature. ${ }^{30}$ A copy of the Tweet with accompanying graphic is presented in figure 1.

\section{Search}

The search strategy was developed in collaboration with a research librarian with expertise in systematic review methodology (NM), as this has been found to increase the quality of the search strategy. ${ }^{31}$ The search included relevant search terms specific to paramedic-related literature. $^{32}$ The search strategy for MEDLINE is presented in table 1 . The full search strategy for the remaining databases is found in online supplementary file 2 .

Table 1 Search strategy for Medline (Ovid interface)

\begin{tabular}{|c|c|}
\hline$\#$ & Searches \\
\hline 1 & $\begin{array}{l}\text { meningococcal infections/ or meningitis, } \\
\text { meningococcal/ or exp Neisseria meningitidis/ }\end{array}$ \\
\hline 2 & (meningococc* or meningitis or neisseria).tw,kf. \\
\hline 3 & or/1-2 \\
\hline 4 & exp Emergency Medical Services/ \\
\hline 5 & Emergency Medical Technicians/ \\
\hline 6 & $\begin{array}{l}\text { (emergency medical service* or EMS or emergency } \\
\text { medical technician* or EMT or emergency service* or } \\
\text { prehospital or pre-hospital or preclinical or pre-clinical } \\
\text { or ambulance* or out-of-hospital or paramedic* or first } \\
\text { responder or HEMS or field triage).tw,kf. }\end{array}$ \\
\hline 7 & or/4-6 \\
\hline 8 & and $/ 3,7$ \\
\hline
\end{tabular}




\section{Selection of sources of evidence}

Selection of sources of evidence for inclusion was determined by two independent reviewers (JP and RP) in accordance with the review eligibility criteria, using the web-based systematic review software, Covidence. ${ }^{33}$ All potentially relevant citations were retrieved in full text for assessment against the inclusion criteria prior to inclusion.

\section{Data charting process}

An initial data charting table was developed to extract information relevant to the review objective. The charting process was iterative, with the table piloted on a subset of relevant sources of evidence and refined further during the data extraction process. The final version of the charting table form is attached as online supplementary file 3. To ensure feasibility of the review, one reviewer (JP) extracted all the data, and the results of each extraction were verified by members of the review group.

\section{Data items}

Data extracted included the characteristics of the literature (eg, type of source, year of publication, country of origin), and key concepts regarding identification and management of IMD by EMS clinicians (eg, antibiotics, risk factors, signs/symptoms). The frequency of words in the included sources (eg, meningitis, penicillin) was extracted from the 'black' literature English language sources, and are presented as a word cloud. The word cloud includes only meaningful words which appeared more than 20 times (ie, words such as 'and' have been removed), and certain related words have been combined (eg, 'pre-hospital' and 'prehospital').

\section{Critical appraisal of individual sources of evidence}

A formal quality appraisal of included literature was not undertaken in this review. This is consistent with the literature regarding the conduct of scoping reviews. ${ }^{8} 1314$

\section{Presentation of results}

The process for the selection of sources of evidence is presented as a flowchart adapted to the requirements of a scoping review from the PRISMA flowchart, ${ }^{34}$ accompanied by a narrative description of the process. The characteristics of the sources of evidence are presented as a narrative and summarised in tabular form with simple descriptive statistics (frequency and percentage). Results of individual sources of evidence are presented in tabular form, and because there were a limited number of sources, are presented as a narrative in chronological order of publication. Results in relation to the objective of the review are presented as a narrative, with tables and graphics (including a word cloud) to aid in their interpretation and to support the narrative.

\section{Patient and public involvement}

While patients were not directly involved with this study, due to the high morbidity and mortality associated with

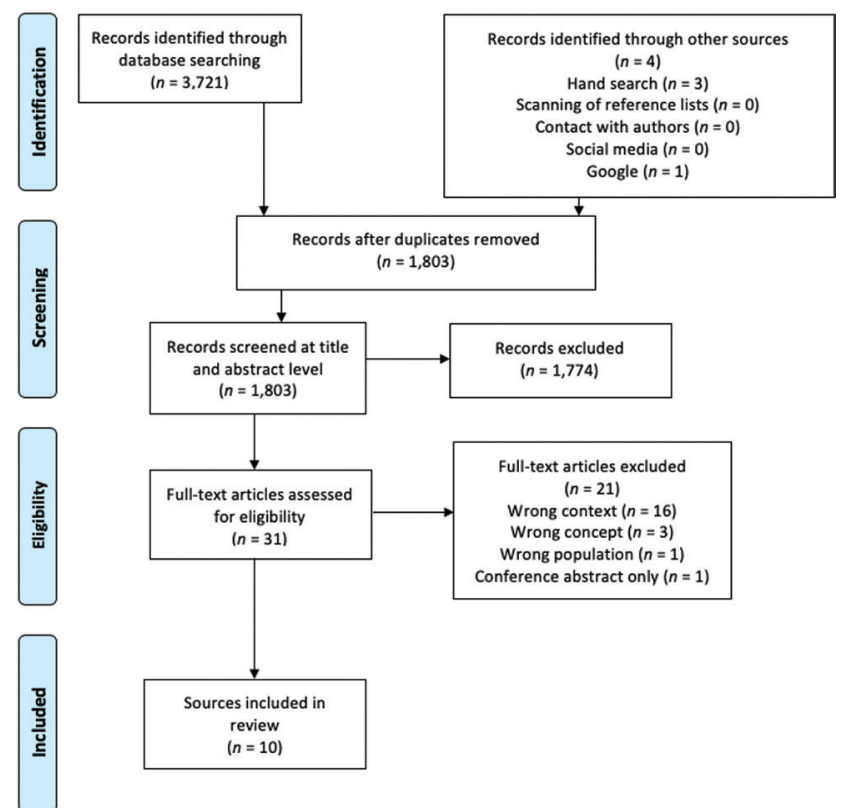

Figure 2 Preferred Reporting Items for Systematic Reviews and Meta-Analyses flow diagram.

IMD, the review authors feel it is a subject of high priority for research.

\section{RESULTS}

\section{Selection of sources of evidence}

Per figure 2, there were 3721 records identified through electronic database searching. There were four records identified through the other sources. After 1923 duplicate records were removed, there were a total of 1803 records available for screening. In all, 1774 records were initially excluded as title and abstract screening did not meet the review eligibility criteria. The full texts of 31 articles were assessed for eligibility. In all, 21 were excluded from the review, with the reasons detailed in figure 2. One located source was a conference abstract from the 13th European Congress of Clinical Microbiology and Infectious Diseases. Details have been reported in online supplementary file 4, but not included in the review's results as abstracts are not yet peer reviewed and have been found to frequently be inconsistent with final manuscripts. ${ }^{35}$ A total of 10 sources of evidence were included in this review.

\section{Social media search results}

In the 1-month period after posting on 9 January 2019, the tweet had received 8846 impressions, 210 engagements, 32 retweets and 26 likes. No additional sources of evidence were located through this method.

\section{Characteristics of sources of evidence}

The characteristics of the included sources of evidence are presented in table 2 . There were a total of 10 sources of evidence. Nine sources of evidence were published journal articles and are considered 'black' literature, while one source was considered 'grey'/unpublished literature. 
Table 2 Characteristics of included sources of evidence

\begin{tabular}{|c|c|c|}
\hline Characteristic & Details & No. $(\%)$ of sources \\
\hline \multirow[t]{2}{*}{ Type of source } & 'Black' literature (Journal article) & $9(90)$ \\
\hline & 'Grey' literature & $1(10)$ \\
\hline \multirow{2}{*}{$\begin{array}{l}\text { Year of } \\
\text { publication }\end{array}$} & 2005-2009 & $6(60)$ \\
\hline & 2010-2014 & $3(30)$ \\
\hline \multirow{4}{*}{$\begin{array}{l}\text { Author country } \\
\text { of origin }\end{array}$} & Australia & $3(30)$ \\
\hline & Czech Republic & $1(10)$ \\
\hline & Germany & $1(10)$ \\
\hline & UK & $4(40)$ \\
\hline $\begin{array}{l}\text { Country income } \\
\text { classification* }\end{array}$ & High-income economies & $10(100)$ \\
\hline \multirow{4}{*}{ Publication } & Der Anaesthesist & $1(10)$ \\
\hline & Journal of Paramedic Practice & $3(30)$ \\
\hline & Klinická mikrobiologie a infekční lékařství & $1(10)$ \\
\hline & Other & $1(10)$ \\
\hline
\end{tabular}

${ }^{*}=$ As defined by the World Bank ${ }^{36}$.

Of the 'black' literature, one source was a proposed treatment protocol, one was a clinical audit and seven were narrative reviews/commentaries/reports. None of these sources raised or answered a specific research question/ aim/objective, and all lacked a rigorous methodological approach and so were not considered original or secondary research studies. All sources were published between 2005 and 2014. The corresponding authors of all sources were based in countries classified as 'highincome' economies by the World Bank. ${ }^{36}$ The majority of sources are published in English in the Australia/New Zealand based Australasian Journal of Paramedicine (4) or the UK-based Journal of Paramedic Practice (3), with one source published in Germany, and one in the Czech Republic.

\section{Results of individual sources of evidence}

The results of individual sources of evidence are presented in table 3. Rožnovský et $a l^{37}$ proposed a standard care protocol for EMS care of IMD in the Czech Republic. The protocol focused on IMD presenting as septicaemia and suggests that blood samples for culture and PCR be obtained prior to administration of an antibiotic as long as it does not delay treatment. The protocol requires the patient to be febrile with a rash plus another symptom to receive active treatment for IMD. The main concept identified in the source was regarding prehospital administration of an antibiotic for meningococcal septicaemia, with the suggested antibiotic being Cefotaxime.

A clinical audit by Cooke ${ }^{38}$ described the prehospital administration of the antibiotic benzylpenicillin by paramedics for meningococcal septicaemia, bench-marked against compliance with the Joint Royal Colleges Ambulance Liaison Committee (JRCALC) clinical guideline, introduced in the UK in 2000. The National Institute for Clinical Excellence defines clinical audit as a quality improvement process that seeks to improve patient care and outcomes through systematic review of care against explicit criteria. ${ }^{39}$ As defined by the Health Research Authority, clinical audit is not considered research. ${ }^{40}$ With the caveat of a limited sample size $(n=69)$, and several methodological problems, the audit found a $78.3 \%$ compliance rate with the JRCALC guideline. While the findings may not adequately reliable to inform practice, they do form a significant proportion of the evidence on the topic. The main concept was that early antibiotic therapy can potentially reduce the burden of disease from IMD.

The narrative review/report by Tippett and Bonham ${ }^{41}$ described the decision-making process of the Queensland Ambulance Service (QAS) in Australia regarding the introduction of an antibiotic into prehospital practice for patients presenting with suspected meningococcal septicaemia. Although the authors acknowledged the concept that prehospital administration of an antibiotic for meningococcal septicaemia can potentially reduce the burden of disease from this condition, they concluded that the low incidence of IMD cases in Queensland, the lack of data on the number of cases responded to by QAS and cost-benefit concerns led them not to introduce an antibiotic agent into clinical practice for patients presenting with suspected meningococcal septicaemia in 2005. 


\begin{tabular}{|c|c|c|}
\hline Author & Type of article & Main concept \\
\hline Rožnovský (2002) $)^{37}$ & Proposal of care protocol & Proposal of care protocol for meningococcal septicaemia \\
\hline Cooke $(2005)^{38}$ & Clinical audit & $\begin{array}{l}\text { Audit of prehospital administration of antibiotic for meningococcal } \\
\text { septicaemia }\end{array}$ \\
\hline Tippett $(2005)^{41}$ & Narrative review/report & $\begin{array}{l}\text { Consideration of introduction of prehospital administration of antibiotic for } \\
\text { meningococcal septicaemia }\end{array}$ \\
\hline Walker (2005) $)^{42}$ & Narrative review/report & $\begin{array}{l}\text { Report on prehospital administration of antibiotic for meningococcal } \\
\text { septicaemia }\end{array}$ \\
\hline Burgess $(2006)^{43}$ & $\begin{array}{l}\text { Narrative review/ } \\
\text { commentary }\end{array}$ & $\begin{array}{l}\text { Social and environmental risk factors to consider prehospital for } \\
\text { identification of meningococcal disease }\end{array}$ \\
\hline Mundy $(2006)^{44}$ & Report & $\begin{array}{l}\text { Prehospital administration of antibiotic for meningococcal septicaemia by } \\
\text { paramedics }\end{array}$ \\
\hline Wiese $(2008)^{45}$ & $\begin{array}{l}\text { Case report and } \\
\text { commentary }\end{array}$ & $\begin{array}{l}\text { Management of meningococcal septicaemia focussing on Waterhouse- } \\
\text { Friderichsen Syndrome }\end{array}$ \\
\hline Glennie $(2011)^{46}$ & Commentary & $\begin{array}{l}\text { Identification and management of meningococcal septicaemia by } \\
\text { paramedics }\end{array}$ \\
\hline Hodkinson $(2013)^{49}$ & $\begin{array}{l}\text { Narrative review/ } \\
\text { commentary }\end{array}$ & Pathophysiology and management of meningococcal disease \\
\hline Valenti $(2014)^{50}$ & $\begin{array}{l}\text { Narrative review/ } \\
\text { commentary }\end{array}$ & Prehospital assessment and treatment of meningococcal meningitis \\
\hline
\end{tabular}

The narrative review/report by Walker ${ }^{42}$ described the introduction of CPGs which include the antibiotic ceftriaxone for meningococcal septicaemia in Victoria, Australia in 2003. It included a brief 'clinical review' of four early cases treated under the new guideline, and a short case study describing a successful patient outcome after paramedics administered ceftriaxone in the field. Walker highlights that prehospital administration of an antibiotic for meningococcal septicaemia was feasible and allowed early access to recommended care when delays may result in further harm, such as in rural or remote settings. Walker highlighted that the evidence for prehospital antibiotics was inconclusive, and that further research is warranted. The main concept was regarding prehospital administration of an antibiotic for meningococcal septicaemia.

The narrative review/commentary article by Burgess ${ }^{43}$ argued that societal and environmental risk factors such as exposure to cigarette smoke, socioeconomic status, dwelling crowding and indigenous status should be incorporated into relevant CPGs and be considered by paramedics in conjunction with clinical risk factors to identify IMD. Although the concept that early antibiotic therapy can potentially reduce the burden of disease from this condition was discussed, the main concept was regarding societal and environmental risk factors for the development and identification of IMD.

The report prepared by Mundy and Merlin for the Australia and New Zealand Horizon Scanning Network ${ }^{44}$ summarised the literature regarding prehospital administration of antibiotics by paramedics for suspected cases of IMD, and was largely a summary of the Tippett and Bonham ${ }^{41}$ and Walker ${ }^{42}$ sources, and included personal communications with one of the review authors (HG). The main concept was regarding prehospital administration of an antibiotic for meningococcal septicaemia.

The article by Wiese ${ }^{45}$ included a brief case report and a commentary on the EMS management of IMD. The case report described a case of a 4-year-old girl with fulminant meningococcal septicaemia who was responded to by EMS. The patient had extensive resuscitation measures which were unfortunately futile. Wiese and colleagues focus on Waterhouse-Friderichsen Syndrome, a complication of IMD resulting in haemorrhage and dysfunction of the adrenal glands.

Glennie's ${ }^{46}$ commentary article promoted resources developed by the Meningitis Research Foundation (MRF) in conjunction with JRCALC to aid prehospital clinicians in the identification and management of meningococcal septicaemia. The article highlighted 'red flag' signs and symptoms which can be used to identify meningococcal disease early. These red flags were found to be early identifiers of meningococcal infection in the general practice setting in research supported by the $\mathrm{MRF}^{47}$ and a later study. ${ }^{48}$ The concept that early antibiotic therapy can potentially reduce the burden of disease from this condition was mentioned; however, the main concept was regarding early 'red flag' signs and symptoms of IMD, particularly in children and adolescence.

The narrative review/commentary article by Hodkinson $^{49}$ described the pathophysiology and current and potential future management of meningococcal septicaemia with some discussion relating to bacterial meningitis. The article included the concept that early antibiotic therapy can potentially reduce the burden of disease from this condition. However, several other 
important concepts, such as the use of oxygen, fluids, inotropes and steroid replacement therapy were considered. The author highlighted that the presence of a rash may be a late sign of IMD, and proposes that it may be justifiable to administer an antibiotic when meningococcal septicaemia is suspected with no rash present; however, further research is required on this topic.

The final article was a narrative review/commentary student submission by Valenti ${ }^{50}$ which focuses on meningococcal meningitis. This is in contrast to all previous articles which were focused on meningococcal septicaemia or mixed presentations. The article described ambiguities in treatment guidelines for bacterial meningitis in the 2013 UK Ambulance Service CPGs, highlighting the weakness of the binary nature of current CPGs and the difficulty in diagnosing bacterial meningitis in the prehospital environment. The article suggested a screening tool such as the Oostenbrink Clinical Decision Rule for Predicting Bacterial Meningitis Risk in Children with Meningeal Signs ${ }^{51}$ be considered for the prehospital environment. Once again, the underlying concept was that early antibiotic therapy can potentially reduce the burden of disease from this condition. It also discussed the concepts of various signs and symptoms (such as Kernig's and Brudzinski's signs) to identify IMD, as well as the use of steroids and paracetamol/acetaminophen as treatment adjuncts.

\section{Synthesis of results}

Table 4 highlights the key concepts present in each of the sources. Most sources focused on patients presenting with septicaemia rather than meningitis. Most sources discussed signs and symptoms to identify IMD, with rash being the most commonly discussed. Interestingly, only two specifically mentioned the 'tumbler test', a method to determine if a rash is non-blanching. Little attention was given to other signs or symptoms, such as painful or cold limbs which has been recognised as an early symptom of IMD. ${ }^{48}$ Only one article focused on risk factors for the development and identification of IMD, with the majority focused on clinical signs and symptoms.

'Early antibiotic therapy potentially reduces the burden of disease from this condition' was the dominant concept across the literature, with all sources of evidence discussing this concept in some detail. Several sources discussed management options other than antibiotics for IMD, and these included the use of expansion fluids, vasopressors and pharmacological adjunctive therapies such as steroids and paracetamol.

Concepts regarding prehospital use of antibiotics for IMD are presented in table 5. The most commonly discussed antibiotic was benzyl penicillin, with the thirdgeneration cephalosporins ceftriaxone and cefotaxime also discussed as potential treatment options. Three sources discussed the concept that administering antibiotics may promote release of endotoxins (endotoxin shower), resulting in clinical deterioration of the patient. Five sources discussed the potential for an anaphylactic reaction after administration of an antibiotic, and two sources presented the concept of the development of penicillin resistance in meningococci.

Figure 3 is a word cloud which presents the frequency of words used in the included English language 'black' literature sources, which can be used as a proxy indicator of the key concepts present. As expected, 'meningococcal' (197), 'disease' (171), 'septicaemia' (91) and 'meningitis' (89) make up four of the six most common words, along with 'patient' (131) and 'case' (108). Next is 'ambulance' (88), then 'penicillin' (84) and 'administer' (82). If 'penicillin' (84) and 'antibiotic' (67) are combined, they become the third most frequent entity (151), highlighting the prominence of the prehospital antibiotics concept.

The only specific sign or symptom included in the word cloud is 'rash', highlighting the frequency that this clinical sign was mentioned in the included sources. Penicillin/antibiotic is the only specific management option discussed with high frequency, highlighting a potential gap in the literature.

\section{DISCUSSION}

\section{Summary of evidence}

In this scoping review, 10 sources of evidence were identified that fit the inclusion criteria. None of these source of evidence raises or answers a specific research question/ aim/objective, and all lacked a rigorous methodological approach and so are not considered primary or secondary research. Because of the difficulties in studying rare diseases, especially in the prehospital environment, and although the included literature reviews/commentaries are inherently biassed due to their methodology, ${ }^{52} 53$ they do present a type of 'text and opinion evidence' (or non-research evidence), and should not be immediately discounted. ${ }^{17}$ Currently, they represent a significant portion of the best available evidence.

Most sources focused on the patient presenting with meningococcal septicaemia. This is indicative of the critical nature of this condition, compared with meningitis presentations which are urgent, but less so than septicaemia. Some of the sources use the terms 'meningitis' and 'meningococcal disease' interchangeably. This has been identified as an issue in the international literature, and it is vital that authors use the correct terminology. ${ }^{54}$

'Early antibiotic therapy potentially reduces the burden of disease from this condition' was identified as the dominant concept across the literature, with all sources of evidence discussing this concept in some detail. We highlight that this concept is credible, but not irrefutable, as the seminal antibiotic studies can be considered inconclusive due to their inherent biases. The other central concept was regarding a rash being used in the identification and to direct active treatment of IMD. The overreliance of the rash as the main sign/symptom to identify IMD and direct treatment is a perceived weakness, and the reviewers suggest that this may be introducing a 'false dilemma', or 'black or white' logical fallacy into clinical 


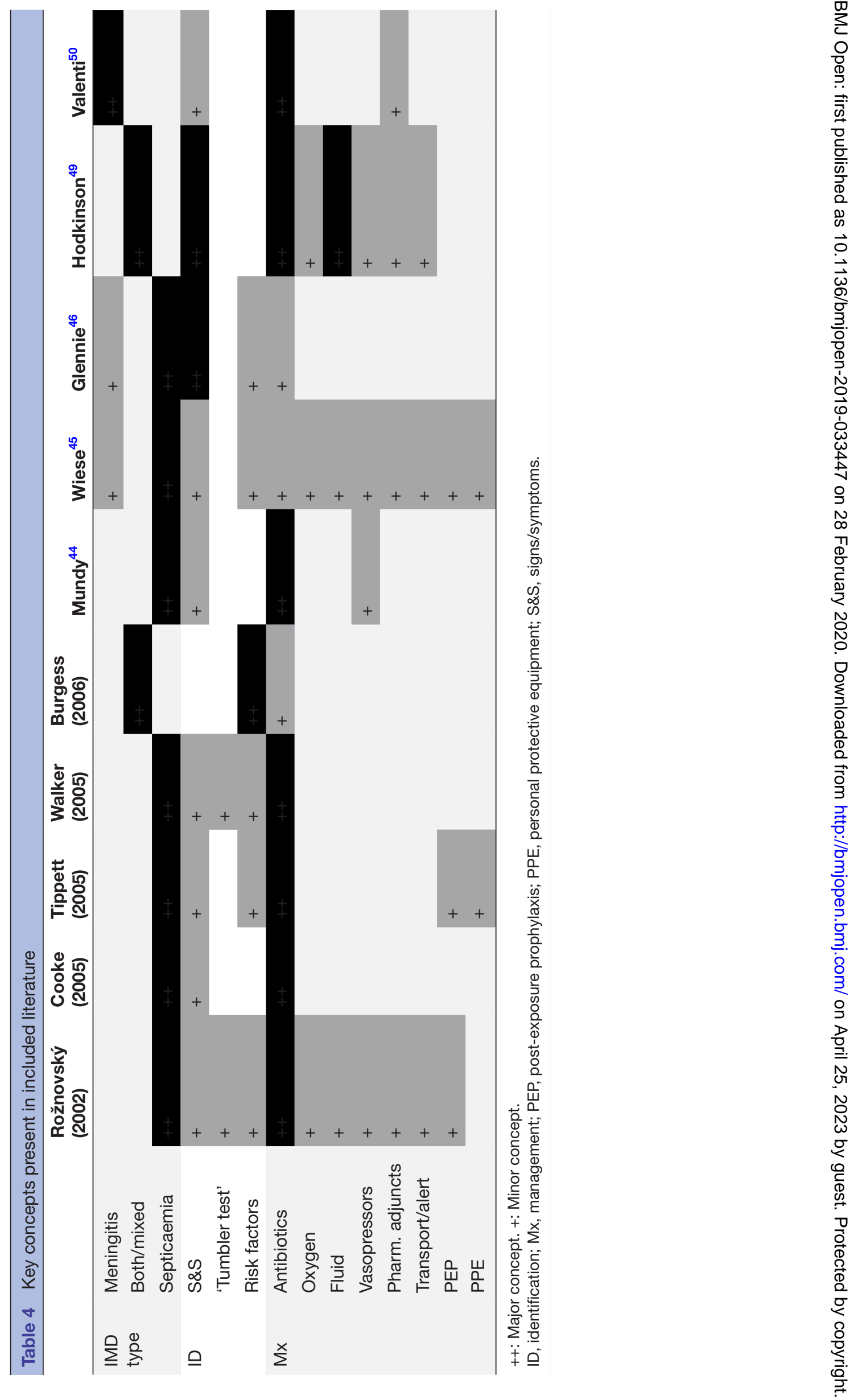




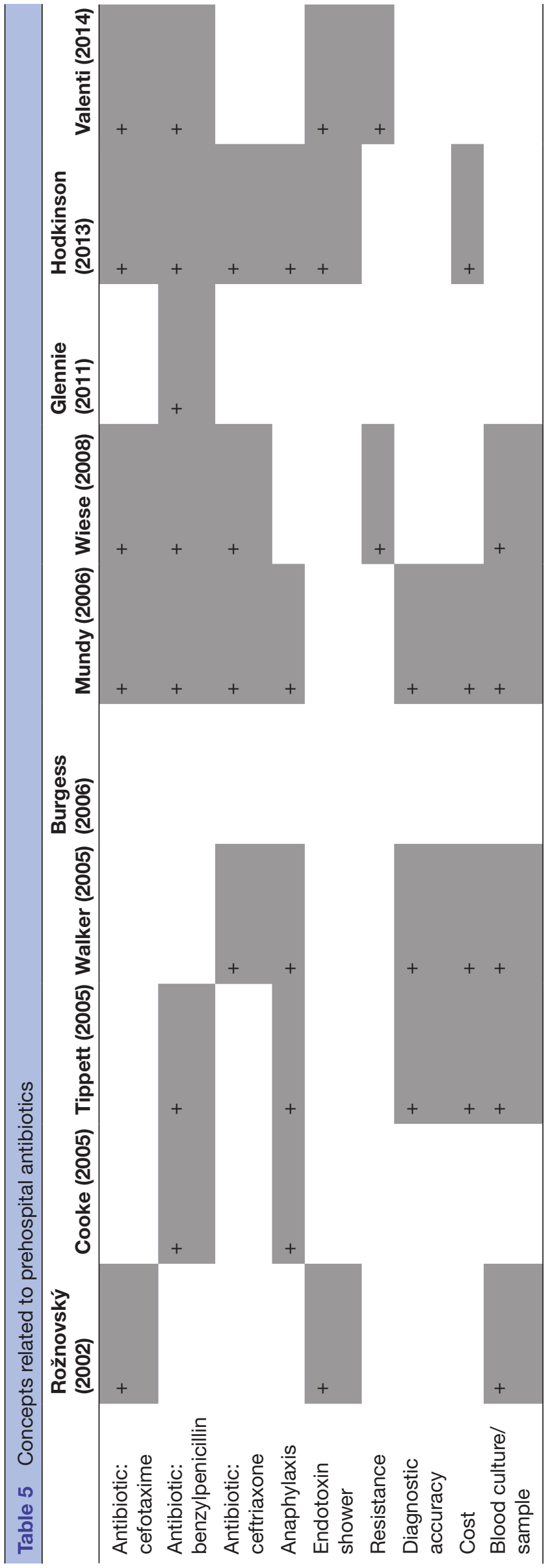

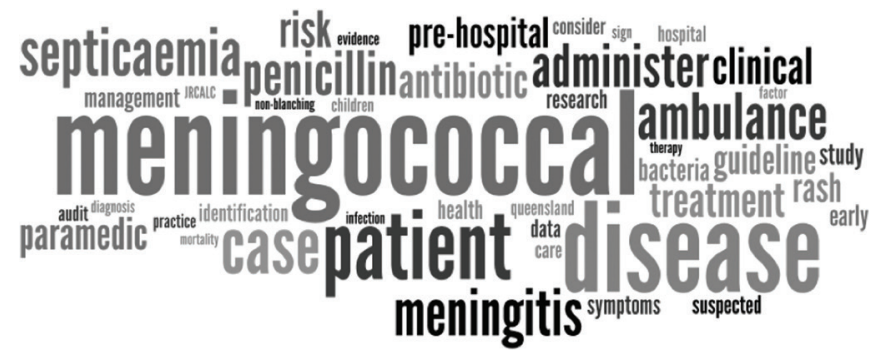

Figure 3 Word cloud demonstrating frequency of words in included sources.

practice, when more nuanced and less binary decisionmaking tools, such as a screening tool, may be more appropriate.

\section{LIMITATIONS}

As with any systematic review, scoping reviews have some limitations. While the review team attempted to perform a highly sensitive search to capture all possible sources of evidence fitting the inclusion criteria, the search strategy may not have located all sources. We attempted to locate relevant 'grey' literature; however, we acknowledge that by definition, it is difficult to comprehensively search for and locate these sources of evidence and some may have been missed. To ensure feasibility of the review, only one reviewer (JP) extracted all the data, and the results of each extraction were verified by the review group. With additional resources, we would ideally have two independent reviewers extract all data and calibrate the extraction process. Finally, we did not include formal methodological appraisal of the included sources. As the level of evidence of the included sources did not rise above 'text and opinion' and we have acknowledged the weaknesses of this type of evidence, we feel that omitting methodological appraisal was appropriate. It is important to recognise that all included sources were from highincome countries, and that lower-income countries may have different priorities for care including peri-natal care and trauma.

\section{CONCLUSIONS}

The state of evidence regarding the care of the patient with IMD by EMS clinicians in the international literature is limited, with no primary or secondary research published in the field. Current evidence relies on clinical audit and text and opinion sources. The dominant concept in the sources is that early antibiotic therapy potentially reduces the burden of disease from this condition.

The sources presume homogeneity in the population in regard to risk factors for developing IMD. The review authors advocate for the consideration of both clinical and social/environmental risk factors in identifying patients with IMD.

There is limited evidence on which to base clinical practice currently. Because of the paucity of evidence 
in the field, reviewing evidence from allied disciplines (eg, medicine) and similar clinical settings (eg, family/ general practice, emergency department, intensive care/ therapy unit) should be undertaken. It is important to recognise that findings from these associated areas are not directly generalisable to the EMS setting due to many factors including heterogeneity in the characteristics of the clinicians (eg, qualifications and skills), healthcare setting (eg, access to diagnostic investigations and assessment conditions) and IMD disease process (eg, emergency call in response to a critically unwell patient). Evidence may also be found in research pertaining to sepsis/meningitis of other aetiologies. It is important to recognise that IMD is caused by a different organism with vastly different characteristics and a unique natural history and so this evidence also has limited generalisability.

Methodologically, rigorous consensus-based guidelines (eg, NICE ${ }^{55}$ and the Surviving Sepsis Campaign ${ }^{56}$ ) represent syntheses of the available evidence along with expert opinion and should direct clinical practice. This review forms the first stage of a research project which will involve observational studies to bridge the gaps in knowledge to support best practice.

\section{Author affiliations}

${ }^{1}$ College of Medicine and Public Health, Flinders University, Bedford Park, South Australia, Australia

${ }^{2}$ South Australian Ambulance Service, Adelaide, South Australia, Australia ${ }^{3}$ Rosemary Bryant A0 Research Centre, School of Nursing and Midwifery, University of South Australia, Adelaide, South Australia, Australia

${ }^{4}$ SA Health Library Service, Bedford Park, South Australia, Australia

${ }^{5}$ School of Medicine and Robinson Research Institute, The University of Adelaide, Adelaide, South Australia, Australia

${ }^{6}$ Vaccinology and Immunology Research Trials Unit, Discipline of Paediatrics, Women's and Children's Hospital Adelaide, North Adelaide, South Australia, Australia

${ }^{7}$ Faculty of Health Sciences, School of Nursing, Midwifery and Paramedicine, Curtin University, Perth, Western Australia, Australia

Acknowledgements The review group would like to acknowledge the assistance of the secondary reviewer Mr. Robin Pap, and article translators Mr. Robin Pap (German), Dr. Jana Merhautová (Czech), Mrs. Hélène Sobolewski (French) and Mrs. Ekaterina Ivanova (Russian).

Contributors JP is the study guarantor and conceived the study, developed the search strategy, assisted in the searches, screened the studies, extracted and interpreted the data and drafted and revised the manuscript. MP contributed towards the design of the study and critically reviewed and revised the draft manuscript. NM assisted in developing the search strategy, performed the searches and assisted in manuscript revisions. $\mathrm{HM}, \mathrm{CH}$ and $\mathrm{HG}$ contributed towards the design of the study, interpretation of the data and edited the draft manuscript. All authors read and approved the final manuscript.

Funding JP receives an Australian Government Research Training Program Scholarship and was partly financially supported by a National Health and Medical Research Council PhD scholarship through the Pre-hospital Emergency Care Australia New Zealand (PEC-ANZ) Centre for Research Excellence (ID:1116453). Funding had no influence on this manuscript.

Competing interests MP declares that he is co-author of the PRISMA Extension for Scoping Reviews (PRISMA-SCR) paper, and that he is the Chair of the Joanna Briggs Institute Methodology Group for Scoping Reviews. All other authors declare that there are no known conflicts of interest.

Patient consent for publication Not required.

Provenance and peer review Not commissioned; externally peer reviewed.
Data availability statement The data analysis of included sources can be accessed upon request by emailing the corresponding author (james.pearce@ flinders.edu.au).

Open access This is an open access article distributed in accordance with the Creative Commons Attribution Non Commercial (CC BY-NC 4.0) license, which permits others to distribute, remix, adapt, build upon this work non-commercially, and license their derivative works on different terms, provided the original work is properly cited, appropriate credit is given, any changes made indicated, and the use is non-commercial. See: http://creativecommons.org/licenses/by-nc/4.0/.

\section{ORCID iDs}

James Pearce http://orcid.org/0000-0001-9580-2943

Helen Marshall http://orcid.org/0000-0003-2521-5166

\section{REFERENCES}

1 Pace D, Pollard AJ. Meningococcal disease: clinical presentation and sequelae. Vaccine 2012;30 Suppl 2:B3-9.

2 Pearce J, Peters MDJ, May N, et al. Care of the patient with invasive meningococcal disease by emergency medical service clinicians: a scoping review protocol. Aus J Paramed 2019;16.

3 Hahné SJM, Charlett A, Purcell B, et al. Effectiveness of antibiotics given before admission in reducing mortality from meningococcal disease: systematic review. BMJ 2006;332:1299-303.

4 Sudarsanam TD, Rupali P, Tharyan P, et al. Pre-Admission antibiotics for suspected cases of meningococcal disease. Cochrane Database Syst Rev 2017;6:CD005437.

5 Smyth MA, Brace-McDonnell SJ, Perkins GD. Identification of adults with sepsis in the prehospital environment: a systematic review. BMJ Open 2016;6:e011218.

6 Smyth MA, Brace-McDonnell SJ, Perkins GD. Impact of prehospital care on outcomes in sepsis: a systematic review. West J Emerg Med 2016;17:427-37.

7 Lane D, Ichelson RI, Drennan IR, et al. Prehospital management and identification of sepsis by emergency medical services: a systematic review. Emerg Med J 2016;33:408-13.

8 Tricco AC, Lillie E, Zarin W, et al. PRISMA extension for scoping reviews (PRISMA-ScR): checklist and explanation. Ann Intern Med 2018;169:467.

9 Khalil H, Peters M, Godfrey CM, et al. An evidence-based approach to scoping reviews. Worldviews Evid Based Nurs 2016;13:118-23.

10 Arksey H, O'Malley L. Scoping studies: towards a methodological framework. Int J Soc Res Methodol 2005.

11 Levac D, Colquhoun H, O'Brien KK. Scoping studies: advancing the methodology. Implement Sci 2010;5:69.

12 Daudt HML, van Mossel C, Scott SJ. Enhancing the scoping study methodology: a large, inter-professional team's experience with Arksey and O'Malley's framework. BMC Med Res Methodol 2013;13:48.

13 Peters MDJ, Godfrey CM, Khalil H, et al. Guidance for conducting systematic scoping reviews. Int J Evid Based Healthc 2015;13:141-6.

14 Peters MDJ, Godfrey C, Mclnerney P, et al. Scoping Reviews. In: Aromataris E, Munn Z, eds. Joanna Briggs Institute Reviewer's Manual. The Joanna Briggs/nstitute, 2017. https://reviewersmanual. joannabriggs.org/

15 Shamseer L, Moher D, Clarke M, et al. Preferred reporting items for systematic review and meta-analysis protocols (PRISMA-P) 2015: elaboration and explanation. BMJ 2015;349:g7647.

16 Open Science Framework. OSF home, 2019. Available: https://osf.io/ dashboard [Accessed 5 Aug 2019].

17 McArthur A, Klugárová J, Yan $\mathrm{H}$, et al. Innovations in the systematic review of text and opinion. Int J Evid Based Healthc 2015;13:188-95.

18 Cartwright K, Reilly S, White D, et al. Early treatment with parenteral penicillin in meningococcal disease. BMJ 1992;305:143-7.

19 Cartwright K, Strang J, Gossain S, et al. Early treatment of meningococcal disease. BMJ 1992;305:774.

20 Gossain S, Constantine CE, Webberley JM. Early parenteral penicillin in meningococcal disease. BMJ 1992;305:523-4.

21 Strang JR, Pugh EJ. Meningococcal infections: reducing the case fatality rate by giving penicillin before admission to hospital. BMJ 1992;305:141-3.

22 Sørensen HT, Møller-Petersen J, Krarup HB, et al. Diagnostic problems with meningococcal disease in general practice. J Clin Epidemiol 1992;45:1289-93.

23 Al-Shaqsi S. Models of international emergency medical service (EMS) systems. Oman Med J 2010;25:320-3.

24 Brouwers MC, Kho ME, Browman GP, et al. Agree II: advancing Guideline development, reporting and evaluation in health care. $J$ Clin Epidemiol 2010;63:1308-11. 
25 Bramer WM, Rethlefsen ML, Kleijnen J, et al. Optimal database combinations for literature searches in systematic reviews: a prospective exploratory study. Syst Rev 2017;6:245.

26 Haddaway NR, Collins AM, Coughlin D, et al. The role of Google Scholar in evidence reviews and its applicability to grey literature searching. PLoS One 2015;10:e0138237.

27 Aromataris E, Pearson A. The systematic review: an overview. Am J Nurs 2014

28 Baudard M, Yavchitz A, Ravaud P, et al. Impact of searching clinical trial registries in systematic reviews of pharmaceutical treatments: methodological systematic review and reanalysis of meta-analyses. BMJ 2017;356:j448.

29 Armstrong R, Jackson N, Doyle J, et al. It's in your hands: the value of handsearching in conducting systematic reviews of public health interventions. J Public Health 2005.

30 @James_W_Pearce. Can you help?2019. Available: https://twitter. com/James_W_Pearce/status/1082881536670588928 [Accessed 9 Feb 2019].

31 Rethlefsen ML, Farrell AM, Osterhaus Trzasko LC, et al. Librarian co-authors correlated with higher quality reported search strategies in general internal medicine systematic reviews. J Clin Epidemiol 2015;68:617-26.

32 Olaussen A, Semple W, Oteir A, et al. Paramedic literature search filters: optimised for clinicians and academics. BMC Med Inform Decis Mak 2017;17:146.

33 Covidence. World-class systematic review management., 2018. Available: https://www.covidence.org/home [Accessed 5 Aug 2019].

34 Moher D, Liberati A, Tetzlaff J, et al. Preferred reporting items for systematic reviews and meta-analyses: the PRISMA statement. PLoS Med 2009.

$35 \mathrm{Li} \mathrm{G}$, Abbade LPF, Nwosu I, et al. A scoping review of comparisons between Abstracts and full reports in primary biomedical research. BMC Med Res Methodol 2017;17:181.

36 World Bank. World bank country and lending groups, 2018. Available: https://datahelpdesk.worldbank.org/knowledgebase/articles/906519world-bank-country-and-lending-groups [Accessed 4 Oct 2018]

37 Rožnovský L, Gutvirth J, Beneš J, et al. Návrh standardu efektivní klinické péče $V$ přednemocniční neodkladné péči (PNP): Invazivní meningokoková onemocnění. Klin Mikrobiol Infekc Lek 2002.

38 Cooke M. Prehospital administration of benzyl penicillin by Paramedics in the UK. Aus J Paramed 2015;3.

39 National Institute for Clinical Excellence. Principles of best practice in clinical audit. Oxon: Radcliffe Medical Press, 2002. https://www.nice. org.uk/media/default/About/what-we-do/Into-practice/principles-forbest-practice-in-clinical-audit.pdf

40 Health Research Authority. Defining research table, 2017. Available: http://www.hra-decisiontools.org.uk/research/docs/DefiningRese archTable_Oct2017-1.pdf
41 Tippett V, Bonham R. Review of the evidence for prehospital administration of benzyl penicillin in meningococcal septicaemia experience in Queensland. Aus J Paramed 2015;3.

42 Walker T. Pre-Hospital paramedic administration of ceftriaxone for suspected meningococcal septicaemia in Victoria, Australia. Aus J Paramed 2015;3.

43 Burgess S. Social and environmental influences affecting the risk of development of meningococcal disease: considerations for prehospital care. Aus J Paramed 2015;4

44 Mundy L, Merlin T. Pre-Hospital administration of antibiotics by paramedics for suspected cases of meningococcal disease., 2006. Available: http://www.horizonscanning.gov.au/internet/ horizon/publishing.nsf/Content/84C1091198F8C1CFCA2575AD 0080F357/\$File/Prehospital admin of antibiotics Vol 14 No 6 Sept2006.pdf.

45 Wiese CHR, Roessler M, Bartels U, et al. Foudroyante Meningokokkensepsis Im Notarztdienst. Anaesthesist 2008;57:369-73.

46 Glennie L. Meningococcal septicaemia: identification and management by paramedics.. J Paramed Pract. 2011.

47 Thompson MJ, Ninis N, Perera R, et al. Clinical recognition of meningococcal disease in children and adolescents. The Lancet 2006;367:397-403

48 Haj-Hassan TA, Thompson MJ, Mayon-White RT, et al. Which early 'red flag' symptoms identify children with meningococcal disease in primary care? Br J Gen Pract 2011;61:e97-104.

49 Hodkinson M. Understanding the process and treatment of meningitis and meningococcal disease. J Paramed Pract 2013.

50 Valenti P. Pre-Hospital assessment and treatment of meningococcal meningitis. J Paramed Pract.2014.

51 Oostenbrink R, Moons KGM, Donders ART, et al. Prediction of bacterial meningitis in children with meningeal signs: reduction of lumbar punctures. Acta Paediatr 2007;90:611-7.

52 Green BN, Johnson CD, Adams A. Writing narrative literature reviews for peer-reviewed journals: secrets of the trade. J Chiropr Med 2006.

53 Grant MJ, Booth A. A typology of reviews: an analysis of 14 review types and associated methodologies. Health Info Libr J 2009;26:91-108.

54 Hodgetts TJ, Brett A, Castle N. The early management of meningococcal disease. Emerg Med J 1998

55 National Institute for Health and Clinical Excellence (NICE). Meningitis (bacterial) and meningococcal septicaemia in under 16S: recognition, diagnosis and management, 2010. Available: https:// www.nice.org.uk/guidance/cg102 [Accessed 13 May 2018].

56 Dellinger RP, Levy MM, Rhodes A, et al. Surviving sepsis campaign: international guidelines for management of severe sepsis and septic shock, 2012. Intensive Care Med 2013;39:165-228. 